\title{
Collagenous Colitis with Endoscopic Manifestations: About a Clinical Case
}

\section{Miguel Vanterpool Hector ${ }^{1 *}$, Yunier Acosta Hernandez ${ }^{2}$ and Odalys Pacheco Sasplugas ${ }^{1}$}

${ }^{1}$ Princess Marina Hospital, Gaborone, Botswana

${ }^{2}$ Guido Valadares National Hospital, Dili, East Timor, Timor-Leste

*Corresponding Author: Miguel Vanterpool Hector, Princess Marina Hospital, Gaborone, Botswana.
Received: August 28, 2021

Published: October 20, 2021

(C) All rights are reserved by Miguel

Vanterpool Hector., et al.

\begin{abstract}
The case of a 45-year-old female patient with a history of Hypertension, smoker and history of omeprazole consumption for a prolonged period was presented, referring to the Gastroenterology consultation of the Princess Marina Hospital of Gaborone in Botswana for a picture of watery diarrhea of more than 6 months of evolution. Laboratory tests, parasitological study, stool culture, abdominal ultrasound and video colonoscopy with biopsy were performed. Video colonoscopy showed edema with areas of severe erythema and mucosal fragility. The histological diagnosis was a collagenous colitis, a type of microscopic colitis that is characterized by a deposit of subepithelial collagen, long-standing watery diarrhea and colonoscopies that show a normal colonic mucosa in the vast majority of cases. Although most of the patients present a normal endoscopic study, it is no frequent but not uncommon for inflammatory signs detected by endoscopy to appear, so this condition should be included in the differential diagnosis with other inflammatory bowel diseases, constituting the histology the only specific diagnostic exam. Treatment with Budesonide was indicated and the patient is currently in clinical remission and follow-up.
\end{abstract}

Keywords: Collagenous Colitis; Gastroenterology; Endoscopy

\section{Introduction}

The first description of microscopic colitis was made in 1976 by Lindstrôm, who published the case of a patient with chronic watery diarrhea who in the rectal biopsy had an important deposit of subepithelial collagen [1]. But it is not until 1980 when Read and collaborators, introduced the term microscopic colitis to describe a disease characterized by the presence of chronic diarrhea, contrasted radiography of the colon by enema and colonoscopy are normal for most of the patients and the diagnosis is achieved through the performance of biopsies of the colonic mucosa [2].

Microscopic colitis is a chronic inflammatory disease of the colon that frequently causes chronic watery diarrhea that could be accompanied by abdominal pain, nocturnal diarrhea, urgency, and fecal incontinence. These symptoms lead to poor quality of life and increase costs of medical care. Diagnosis is made by histological examination of multiple colonic mucosal biopsy samples, which often demonstrate no or only a few abnormalities in endoscopy. Two main histological subtypes may be collagenous colitis and lymphocytic colitis, but it has been determined that the incomplete form exists with non specific features [3].

The pathogenesis of microscopic colitis is complex and multifactorial. It can include luminal factors, immune dysregulation, and genetic predisposition. Endoscopic inflammatory changes are increasing in patients with collagenous colitis; however, they are non 
specific. Although patients with normal endoscopy are prevalent, the number of patients with endoscopic manifestations associated with this disease is significant [4].

Recently, the Microscopic Colitis Disease Activity Index (MCDAI) has been proposed, based on the number of liquid stools and the presence of nocturnal stools, abdominal pain, weight loss, urgency and fecal incontinence, a score that is used as a tool to assess activity. The main objective of treatment is to achieve clinical remission and improve the quality of life of the patients [5].

\section{Presentation of the case}

Patient of 45 years of age,female, rural origin, smoker, HIV negative, with a history of Hypertension for which he takes treatment with Amlodipine $5 \mathrm{mg}$ and Hydrochlorothiazide $25 \mathrm{mg}$ a day and Chronic Gastritis for which she self-medicated Omeprazole for a period greater than 8 months, was referred from his primary health service for presenting watery diarrhea without mucus or blood in number of two to three on the day of little to moderate amount and slight abdominal discomfort. No alarm symptoms or signs such as loss of appetite, weight loss or digestive bleeding were reported.

\section{Physical exam}

- Mucous membranes: Normocolored and wet.

- Cardiovascular apparatus: Audible and rhythmic heart sounds. Heart rate: 82 beats per minute.

- Blood pressure: 140/94 mm Hg.

- Respiratory System: Normal vesicular murmur, no rales, Respiratory rate: 19 breaths per minute.

- Abdomen: Flat, which follows respiratory movements, no visceromegaly or palpable tumor, no pain on superficial or deep palpation. Normal hydro-aircraft noises.

- Subcutaneous cell tissue: Not infiltrated.

- Nervous system: Oriented in time, space and person. No neurological focus.

\section{Complementary studies}

Laboratory tests

- Hemoglobin: $14.3 \mathrm{~g} / \mathrm{l}$

- Total leukocytes: $4.68 \times 10^{9} / 1$

- Platelets: $214 \times 10^{9} / 1$
- $\quad$ Sodium: $134 \mathrm{mmol} / \mathrm{l}$

- Potassium: $3.9 \mathrm{mmol} / \mathrm{l}$

- Chlorine: $100.5 \mathrm{mmol} / \mathrm{l}$

- Urea: $3.9 \mathrm{mmol} / \mathrm{l}$

- Creatinine: $81 \mathrm{mmol} / \mathrm{l}$

- GGT: $63.0 \mathrm{U} / \mathrm{L}$

- $\quad$ ASAT: $22 \mathrm{U} / \mathrm{L}$

- TOOLS: $30.7 \mathrm{U} / \mathrm{L}$

- ALP: $57 \mathrm{U} / \mathrm{L}$

- Total proteins: $63.1 \mathrm{~g} / \mathrm{l}$

- Albumin: $40.8 \mathrm{~g} / \mathrm{l}$

- Parasitological stool test: Negative.

- Coprocultive: Negative.

- Occult blood in feces: Negative.

\section{Abdominal ultrasound}

Liver of normal size and echogenicity. Gallbladder: normal size, thin walls. No lithiasis. Normal intrahepatic bile ducts. Normal bile duct. Pancreas of normal size and echogenicity. Normal spleen. Both kidneys of normal size and parenchyma. No lithiasis.

\section{Videocolonoscopy}

(Figure 1): Edematous mucosa with extensive and severe areas of erythema and friability was seen in the cecum, ascending, transverse, descending and sigmoid. Normal rectum.
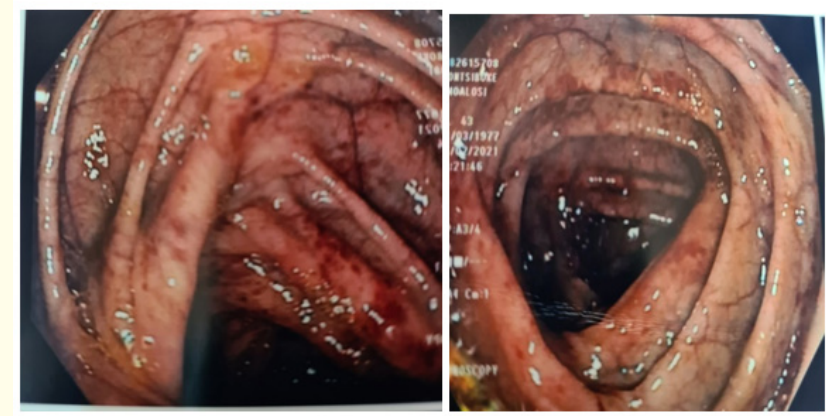

Figure 1: Endoscopy study that shows an edematous mucosa with extensive and severe areas of erythema and friability. 


\section{Colon biopsy}

Separate samples were taken from cecum, transverse, descending and sigmoid.

- Cecum and Sigmoid: A thickened layer of subepithelial collagen is observed. The columnar epithelium is normal but focal areas of ulceration are appreciated. The crypts and glands are normal. Increase of inflammatory cells such as lymphocytes and plasma cells around the glands, crypts and lamina propria.

- Transverse and Descending: A thickened layer of subepithelial collagen is observed. The columnar epithelium looks normal. The crypts and glands are normal. Increase of inflammatory cells such as lymphocytes and plasma cells around the glands, crypts and lamina propria. The lamina propria shows extravasation of erythrocytes (Figure 2).

\section{Histological diagnosis}

Collagenous colitis.

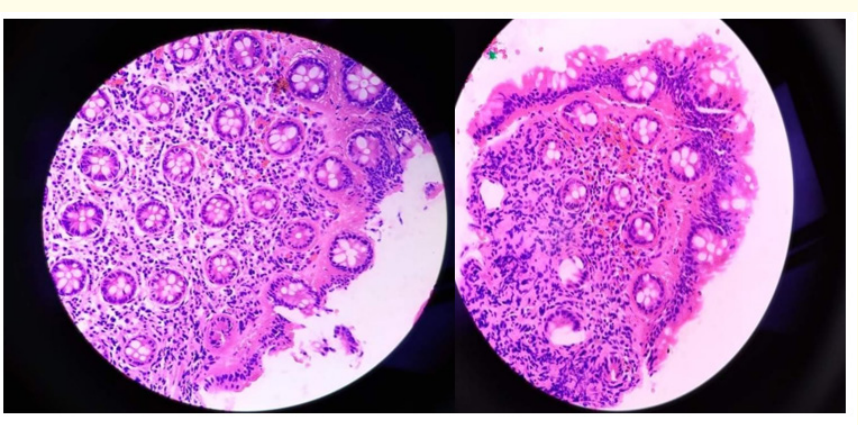

Figure 2: Histological study shows the inflammatory infiltrate and the subepithelial collagen layer.

In the first consultation by Gastroenterology the consumption of Omeprazole was suspended and she was advised to stop smoking. After the complementary exams, and once the histological diagnosis was obtained, treatment with Budesonide was started with a dose of $9 \mathrm{mg}$ per day for two months achieving clinical improvement at ten days and complete clinical remission of the disease in about four weeks. The patient continues to be seen as outpatient at Gastroenterology clinic and she has been maintained asymptomatic.

\section{Discussion and Conclusion}

Microscopic colitis (CM) is a chronic inflammatory bowel disease characterized by non-bleeding diarrhea with normal-looking colonic mucosa and distinct histopathological features. It has a preponderance for middle-aged women and is associated with several autoimmune disorders, as well as widely used medications, including NSAIDs. This disease has two main subtypes, collagenous colitis (CC) and lymphocytic colitis (LC), an also an incomplete variant. Although it has been well described in the medical literature since the 1970s, few studies are available to guide clinical management [6].

The incidence of CC is 4.14 per 100,000 person-years and 4.85 per 100,000 person-years for LC. The prevalence of CC is 49.21 cases per 100,000 person-years and 63.05 cases per 100,000 person-years for LC [7]. It is important to note that most data on the incidence and prevalence of MC come from the developed world, such as the United States, Canada, Spain, the Netherlands and Denmark. There is a lack of data and controlled studies from developing countries. Age increases the risk of MC with an estimated 8.3 times more by age $>65$ years compared to $<65$ years. The incidence is higher in women than in men, with a female-male incidence ratio of 3.05 for CC and 1.92 for LC [8].

The association between drug intake and the development of MC is still controversial. It is known that both proton pump inhibitors (PPIs) and nonsteroidal anti-inflammatory drugs (NSAIDs) can affect colonic permeability, allowing the passage of antigens to the lamina propria which can cause an immune reaction leading to lymphocytosis [9].

Smoking has an established relationship with inflammatory bowel disease and its association with MC has also been recently proposed. In an observational study, $100 \%$ of people with CC had a history of smoking before the development of the disease [10].

Endoscopic findings are most often recognized in patients with MC; however, they are not specific. Initially, it was estimated 20\% of cases with positive endoscopy with non-specific inflammatory process that occurs in the form of edema and erythema of the mucosa, however, currently studies show an increase in its incidence by $38.8 \%$ [11]. 
The histopathological criteria of CC are: a thickened subepithelial collagenous band $\geq 10 \mathrm{~mm}$ combined with an increase in the inflammatory infiltrate in the lamina propria of eosinophils, lymphocytes and plasma cells. The criteria apply to hematoxylin and eosin (HE) study [4].

Guidelines from the American Gastroenterological Association (AGA) recommend budesonide $9 \mathrm{mg}$ daily for eight weeks to induce remission, showing rapid improvement in diarrhea, usually within two weeks. In patients who do not respond to budesonide, it is recommended to search for alternative or associated diagnoses such as celiac disease, associated inflammatory bowel disease or the persistence of the trigger factor. In these patients, the use of immunomodulators such as azathioprine or metrotexate should be considered with variable results [12].

\section{Final Considerations}

Collagenous colitis has been associated over the years with a normal endoscopic pattern. However, the frequency of inflammatory non specific findings in Colonoscopy of variable intensity is increasing nowadays, so it should be included in the differential diagnosis of the spectrum of non specific colitis. The histological study continues to be the definitive diagnosis.

\section{Conflicts of Interest}

The authors declare that they do not present any type of conflict of interest.

\section{Bibliography}

1. Lindstrom CG. "Collagenous colitis" with watery diarrhoea-a new entity?" Pathology European England 11 (1976): 87-89.

2. Muñoz Jackson G. "Microscopic colitis". Revista Médica de Costa Rica y Centroamérica LXXI.611 (2014): 471-476.

3. Miehlke S., et al. "Microscopic colitis: pathophysiology and clinical management". Lancet Gastroenterology and Hepatology 4.4 (2019): 305-314.

4. Miehlke S., et al. "European guidelines on microscopic colitis: United European Gastroenterology and European Microscopic Colitis Group statements and recommendations". United European Gastroenterology Journal 9.1 (2021): 13-37.
5. Cotter TG., et al. "Development of a Microscopic Colitis Disease Activity Index: a prospective cohort study". Gut 67 (2018): 441446.

6. Fernández-Bañares F., et al. "Current concepts on microscopic colitis: evidence-based statements and recommendations of the Spanish microscopic colitis group". Alimentary Pharmacology and Therapeutics 43.3 (2016): 400-426.

7. Tong J., et al. "Incidence, prevalence, and temporal trends of microscopic colitis: a systematic review and meta-analysis". American Journal of Gastroenterology 110.2 (2015): 265-276.

8. Zabana Y., et al. "Advances for improved diagnosis of microscopic colitis in patients with chronic diarrhoea". Gastroenterology and Hepatology 40.2 (2017): 107-116.

9. Gentile N and Yen EF. "Prevalence, pathogenesis, diagnosis, and management of microscopic colitis". Gut Liver 12.3 (2018): 227-235.

10. Vigren L., et al. "Is smoking a risk factor for collagenous colitis?". Scandinavian Journal of Gastroenterology 46 (2011): 1334-1339.

11. Kane JS., et al. "Macroscopic findings, incidence and characteristics of microscopic colitis in a large cohort of patients from the United Kingdom". Scandinavian Journal of Gastroenterology 52 (2017): 988-994.

12. Nguyen GC., et al. "American Gastroenterological Association Institute guideline on the medical management of microscopic colitis". Gastroenterology 150 (2016): 242-246.

\section{Volume 3 Issue 11 November 2021 \\ (C) All rights are reserved by Miguel Vanterpool Hector., et al.}

